\title{
Mobile Proxies for Proactive Buffering in Wireless Internet Multimedia Streaming
}

\author{
Paolo Bellavista, Antonio Corradi, Carlo Giannelli \\ Dip. Elettronica, Informatica e Sistemistica - Università di Bologna \\ Viale Risorgimento, 2 - 40136 Bologna - ITALY \\ Phone: +39-051-2093001; Fax: +39-051-2093073 \\ \{pbellavista, acorradi, cgiannelli\}@deis.unibo.it
}

\begin{abstract}
The widespread popularity of roaming wireless devices with limited and heterogeneous capabilities is enabling new challenging deployment scenarios for multimedia streaming in wired-wireless integrated networks. Streaming services should consider not only the support of different forms of mobility and connectivity, but also runtime service personalization based on client characteristics and location, while possibly maintaining streaming continuity independently of client roaming. The paper proposes an original middleware solution based on mobile proxies that work at the wired-wireless network edges, close to their limited wireless clients, to support their personalized access to continuous services, especially by pre-fetching multimedia contents to avoid streaming interruptions. In particular, the paper focuses on how to exploit handover prediction i) to migrate mobile proxies in advance to the wireless cells where mobile clients are going to reconnect, and ii) to proactively manage mobile proxy buffers, thus reducing memory and bandwidth usage. Experimental results show that our original lightweight solution for IEEE 802.11 cell handover prediction can significantly improve the management of proactive multimedia buffers, by respecting the challenging time constraints of the addressed application domain.
\end{abstract}

\section{Introduction}

Let us consider the common deployment scenario where wireless solutions extend the accessibility to the traditional Internet via Access Points (APs) working as bridges between fixed hosts and wireless devices [1]. The most notable example is the case of IEEE 802.11 APs that support connectivity of Wi-Fi terminals to a wired local area network [2]. We will use the term Wireless Internet (WI) to indicate these integrated networks with fixed Internet hosts, wire- less terminals, and wireless APs in between.

Service provisioning over the WI must dynamically consider the characteristics of currently served client devices, primarily their possible limits on local resources and their high heterogeneity. Limited processing power, memory, and file system make portable wireless devices unsuitable for traditional services designed for fixed networks. These constraints call for both assisting wireless terminals in service access and downscaling service contents depending on terminal resource constraints. In addition, client devices currently exhibit extreme heterogeneity of hardware capabilities, operating systems, installed software, and connectivity technologies. This heterogeneity makes hard to provide all needed service versions with statically tailored contents and calls for on-thefly adaptation of service contents.

Client resource limits and heterogeneity are particularly crucial when providing continuous services, i.e., applications that distribute time-continuous flows of information to their requesting clients, such as in the case of audio and video streaming [3]. WI continuous services should address several challenging issues, from quality management to runtime personalization of streaming contents. A particularly hard task, especially when associated with the above issues, is to avoid temporary flow interruptions when clients roam from one wireless locality to one another, also by considering the often strict limits on client memory, which do not allow traditional buffering solutions based on proactive client caching of large chunks of multimedia flows.

We claim the need of middleware solutions for the provisioning of WI continuous services to portable devices, by locally mediating their access and by dynamically adapting service content to client terminal properties, client location, and runtime resource availability [2-5]. In addition, middleware components should dynamically deploy by following client roaming among wireless localities, in order to locally assist clients during their service sessions. Moreover, 
client memory limitations suggest having middleware components executing on the fixed network, where and when needed, while portable devices should only host thin clients, loaded by need and automatically discarded after service.

By following the above guidelines, we have developed a middleware, based on Secure and Open Mobile Agent (SOMA) proxies, to support locationaware continuous services to wireless devices with strict limits on on-board resources [6, 7]. The primary design idea is to dynamically deploy mobile proxies acting on behalf of wireless clients over the fixed hosts in the network localities that currently offer client connectivity. Mobile proxies hide the complexity of maintaining personalized service sessions (notwithstanding provision-time client roaming) from device clients, which can remain simple and lightweight.

In particular, the paper focuses on an essential aspect of our middleware: how to avoid interruptions of continuous service provisioning when a client roams from one wireless locality to one another (wireless cell handover) at runtime. To achieve this goal, handover prediction is crucial. On the one hand, it permits to migrate mobile proxies in advance to the wireless cells where mobile clients are going to reconnect, so to proactively reorganize user sessions in newly visited network localities. On the other hand, it enables the proactive management of proxy-sided buffers with pre-fetched multimedia streaming content. In fact, exploiting too large proxy buffers uselessly waste the memory of places hosting proxy execution and uselessly overload the network when proxies migrate (together with their state) to wrongly predicted localities. Too small proxy buffers endanger streaming continuity since they rapidly become obsolete after proxy migration. Handover prediction can enable the adaptive management of proxy-sided buffers, by increasing the size of the pre-fetched streaming contents in the buffer (of the amount expectedly needed) only in anticipation of client handovers.

The proxy-sided proactive buffering presented in the paper integrates with a second level of smallersized adaptive buffers maintained by our middleware stubs that wrap application clients at wireless devices. The management of client-sided buffers, also based on wireless cell handover prediction, is out of the scope of the paper and described in [8].

We have thoroughly evaluated the performance of two variants, exploiting two different mobility predictors, of our adaptive buffering solution for mobile proxies. A wide set of experimental results have been measured in a simulated environment, which can model large sets of nodes randomly roaming among IEEE 802.11 APs. In addition, we have collected in- the-field results by deploying our system prototype over a small set of Wi-Fi laptops. The reported results show that both proposed variants outperform traditional buffering solutions based on statically predetermined buffer size: they permit to reduce the buffer size needed to maintain streaming continuity and impose a very limited overhead, by only exploiting monitoring data about Received Signal Strength Indication (RSSI), in a completely decentralized way.

\section{Mobile Proxy-based Middlewares for Continuous Service Provisioning}

On the one hand, WI service provisioning calls for dynamically personalizing service fruition to suit the specific (often limited) characteristics of wireless clients. On the other hand, provision-time device mobility requires additional support operations that are too expensive to be performed by severely limited terminals, e.g., location-dependent resource rebinding.

We claim the need for distributed and active infrastructures of mobile middleware proxies working in the fixed network on behalf of portable devices to mediate their personalized access to WI services. In particular, in continuous services, mobile proxies should negotiate the proper stream quality level depending on terminal characteristics and user preferences, should choose the most suitable streaming server depending on client location, and should monitor, control, and possibly downscale the quality of the received multimedia stream in the case of wireless network congestion. In the WI all these management operations should be performed over the fixed network at the wired-wireless network edges where resource discontinuities are sharper and more frequent. In addition, when clients roam at provision time, their proxies should be able to follow them by migrating to the newly visited wireless localities, to maintain colocation with their served access terminals [7].

According to the above guidelines, we have designed and implemented a SOMA-based middleware for WI continuous services. Our middleware provides any wireless device with one SOMA-based companion entity, called shadow proxy, which runs in a wired node (place) in the same wireless locality that currently provides connectivity to the device [9]. Figure 1 shows that wired/wireless hosts in a locality can be grouped into logical domains; domains are disjointed, even if they include APs with partially overlapping wireless coverage areas.

In this provisioning environment, handover prediction is crucial to avoid service interruptions when serving audio/video streams to roaming clients. 
Handover prediction permits to perform the needed service management operations in advance with regards to the actual communication-level client handover. On the one hand, it enables the anticipated migration of mobile proxies to the next domain of attachment of their associated clients. On the other hand, it makes possible to adapt the size of proxysided buffers with pre-fetched streaming data depending on client handover probability. In particular, the primary guideline is to increase the pre-fetched data in the buffer when the wireless cell handover of the corresponding client is expected to occur soon, while the proxy buffer size is decreased when the predicted probability of a handover in the near future is low. The goal is not only to proactively migrate proxies in the correctly predicted next wireless localities, but also to move them there with the needed buffered data to enable service continuity, while minimizing network traffic due to useless streaming pre-fetching.

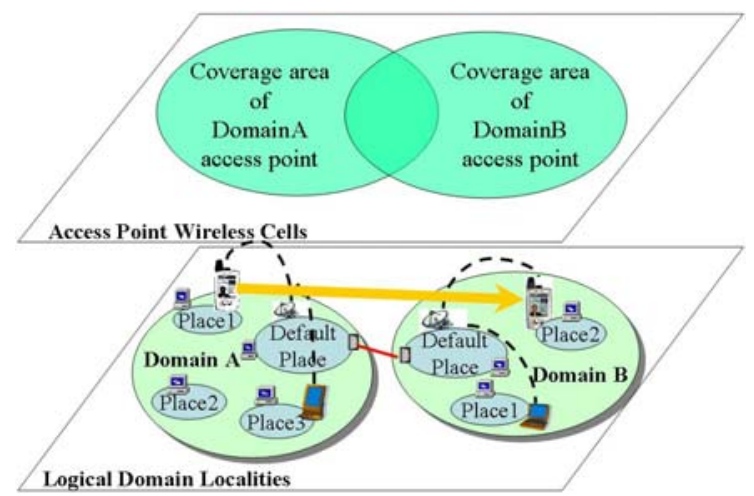

Figure 1. Portable devices roaming among SOMA wireless access localities.

To clarify the motivations for handover prediction, let us consider an actual deployment scenario and rapidly overview the service management operations that the SOMA-based middleware has to perform in response to a client change of locality. Let us suppose a user roams from DomainA to DomainB in Figure 1 while she is receiving her personalized multimedia flow. Depending on the handover strategy of the underlying communication layer (see Section 3.1), the user device is transparently de-associated from the origin wireless cell and associated to the destination one when it either completely loses the origin signal or is still in the overlapping coverage area between the two cells. In any case, before the communicationlevel handover completes, the SOMA-based middleware should migrate the shadow proxy to the destination domain, the proxy should instantiate/configure the needed local middleware components there and should reconnect to the server (or to an equivalent local replica of it), before being capable of serving its client again. Also in presence of a correctly anticipated proxy migration, these operations may produce a temporary streaming suspension if not coupled with smart buffering techniques for adaptive multimedia pre-fetching [7].

A detailed description of the SOMA middleware components for continuous services and of our original RSSI-based solution for wireless cell handover prediction can be found in $[6,7]$. In the following, we specifically focus on the original proposal of this paper, i.e., how to exploit handover prediction for the optimized adaptive management of proxy-sided streaming buffers.

\section{Proactive Management of Proxy-sided Adaptive Buffers}

Given the crucial role of efficient buffer management to prevent streaming interruptions in the case of provision-time client roaming, we propose an innovative buffer management solution that tends to optimize bandwidth and memory utilization by increasing the proxy buffer size only when a client handover is going to occur (and reducing the size when we do not forecast any near handover). Let us observe that our adaptive buffering, specifically developed for our mobile proxy-based middleware to avoid streaming interruptions in continuous services, can help any WI service that benefits from content pre-fetching in client wireless access localities.

The section first clarifies how communicationlevel handover works: IEEE 802.11 does not specify any specific handover strategy and communication hardware manufacturers are free to implement their own strategies. The different communication-level handover strategies implemented in the currently available Wi-Fi equipment motivate different variants of our prediction-based buffer management solution: for this reason, the paper proposes and compares two alternative buffer management implementations, specifically designed for the two most relevant classes of possible handover strategies, i.e., Hard Proactive (HP) and Soft Proactive (SP).

\subsection{Communication-level Handover Strate- gies: Reactive and Hard/Soft Proactive}

Several communication-level handover strategies are possible, which mainly differ in the event used to trigger the handover. It is possible to distinguish between two main strategy categories, reactive and proactive. Reactive handover strategies tend to delay handover as much as possible: handover starts only when wireless clients completely lose their current 
AP signal. Reactive strategies are effective in minimizing the number of handovers, e.g., by avoiding to trigger a handover process when a client approaches a new wireless cell, without losing the origin signal, and immediately returns back to the origin AP. However, reactive handovers tend to be long because they include looking for new APs, choosing one, and asking for re-association. Proactive strategies, instead, tend to trigger handover before the complete loss of origin cell signal, e.g., when the new cell RSSI overpasses the origin one. These strategies are less effective in reducing the number of useless handovers but are prompter, by performing search operations for new APs before the handover procedure starts.

By concentrating on proactive strategies, a further classification is possible. On the one hand, HP strategies trigger a handover any time the RSSI of a visible AP is greater than the RSSI of the currently associated AP plus an Hysteresis Handover Threshold (HHT); HHT is introduced mainly to prevent heavy bouncing effects. On the other hand, SP strategies are "less proactive" in the sense that they trigger handover only if i) the HP condition applies (there is an AP with RSSI greater than current AP RSSI plus HHT), and ii) the current AP RSSI is lower than a Fixed Handover Threshold (FHT).

For instance, the handover strategies implemented by Cisco Aironet 350 and Orinoco Gold Wi-Fi cards follow, respectively, the HP and SP models. More in detail, Cisco Aironet 350 permits to configure its handover strategy with the "Scan for a Better AP" option: if the current AP RSSI is lower than a settable threshold, the Wi-Fi card monitors RSSI data for all visible APs; for sufficiently high threshold values, the Cisco cards behave according to the HP model. Orinoco Gold cards exactly implements the SP strategy, without giving any possibility to configure the used thresholds.

\subsection{The Proxy Buffer Design Guidelines}

The ultimate goal of our prediction-based buffer management is to proactively migrate a proxy to the next client access locality before the actual client handover; pre-fetched data in proxy buffers should grow only immediately before starting migration, so to minimize buffer/bandwidth consumption, as better detailed in the following.

Just to give a rough idea of the proxy buffer size needed in the addressed multimedia scenario, let us consider the simple case of a client receiving a multimedia stream played at $1.0 \mathrm{Mbps}$ constant bitrate, a client-to-proxy bandwidth of $1.5 \mathrm{Mbps}$, and resource rebinding operations after proxy migration taking $2 \mathrm{~s}$ (rebinding interval includes the time for server re- connection and for client-specific service personalization). After handover, the proxy buffer already available at the new access locality should be at least $2 \mathrm{~s} * 1.0 \mathrm{Mbps}=250 \mathrm{~KB}$. Let us note that if handover prediction is too anticipated, migrated proxy buffers become obsolete and useless. Therefore, it is crucial to migrate proxies only when needed and to overestimate buffer size with regards to the minimum $250 \mathrm{~KB}$. In the following, we will consider a maximum buffer size of $800 \mathrm{~KB}$, corresponding to $6.4 \mathrm{~s}$ of pre-fetched streaming content consumed at $1.0 \mathrm{Mbps}$. In the case the average useful bandwidth between wired hosts is $6 \mathrm{Mbps}$ (successive wireless access localities are close), the movement of a full buffer proxy takes about $1.5 \mathrm{~s}$, approximately the same time interval needed for completing communication-level handover in most common Wi-Fi equipment [10].

We have designed and implemented a handover predictor that triggers the growth/reduction of the proxy-sided buffered data and the proxy migration by comparing the predicted RSSI values of both the currently associated AP and all other visible APs (some details about the adopted RSSI prediction model are in Section 3.3). The predictor runs at the client side, is completely decentralized, and only exploits locally available RSSI monitoring data; RSSI awareness is achieved in a completely portable way over heterogeneous platforms [11].

Our adaptive buffering imposes buffer size to be usually low $(200 \mathrm{~KB})$ to save memory at the proxy host and to avoid useless network overhead; in fact, when clients do not change their APs, the buffering goal is only to smooth possible server-to-proxy bandwidth fluctuations. When the predictor notifies a proxy that its associated client is going to change its wireless cell, the proxy sets buffer size to maximum $(800 \mathrm{~KB})$, waits for buffer fulfilment, and then commands the migration of its clone, with the fulfilled buffer, to the predicted location. If the client disassociates from the origin AP before buffer is full, the proxy immediately sends its clone to the predicted location with the already buffered data. After clone migration, the proxy in the origin locality sets buffer size again to minimum and continue serving its client until it leaves the cell. If client entrance in the predicted cell occurs too late with regards to clone migration, part of the migrated buffer becomes obsolete. For this reason, in the case of client not arrived yet, the middleware automatically re-sends an updated buffer to an already predicted location after a time interval equal to buffer duration - buffer fulfilment proxy migration + communication handover (6.4$1.92-1.5+1.5=4.48 \mathrm{~s}$ in the above scenario). 


\subsection{Hard/Soft-Proactive Handover Predic- tors for Proxy-sided Adaptive Buffers}

Our adaptive buffer management solution can exploit two alternative variants of handover predictor, one suitable for communication-level HP handovers and the other for SP ones. We have decided not to implement handover predictors for reactive strategies because reactive handovers are inherently unsuitable for continuous service provisioning, given their longer time needed for handover completion. In addition, handover prediction is less challenging for reactive communication-level handovers than when dealing with proactive ones: the triggering of a reactive handover only depends on one AP RSSI data.

The implemented HP-variant of our handover predictor triggers a prediction when the predicted RSSI value for the current AP is lower than predicted RSSI values for any visible AP plus an Hysteresis Prediction Threshold (HPT). In the case of several predictions simultaneously enabled, the predictor only considers the most probable one (associated with the AP with strongest RSSI) to avoid the proliferation of migrated proxies. Figure 2 depicts predicted RSSI values for the current AP and the next one, in proximity of an HP handover. A wireless client, moving from the origin AP to the destination AP, is first associated with the origin (white background), then with the destination (grey background). When the predicted RSSI of the destination AP overcomes the predicted RSSI of the origin AP plus HHT, the handover is triggered.

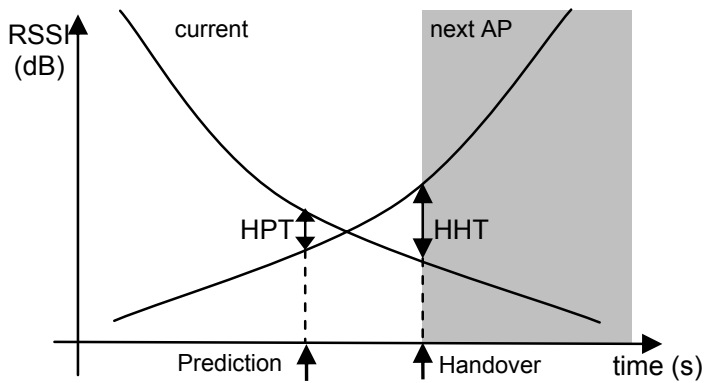

Figure 2. HP-variant prediction and handover triggers.

The implemented SP-variant of the handover predictor triggers a prediction when the predicted RSSI value for the current AP is lower than i) a Fixed Prediction Threshold (FPT) and ii) a predicted RSSI value for one visible AP plus HPT. Similarly to HP, the SP-variant predictor only considers the most probable future locality in the case of several predictions simultaneously enabled. Figures 3 and 4 show predicted RSSI values for the origin and the destination APs in proximity of an SP handover. Figure 3 depicts a case where predicted RSSI values change quite slowly: it is the overcoming of hysteresis thresholds that triggers handover prediction. In Figure 4, instead, predicted RSSI values rapidly evolve, and the passing of fixed thresholds produces handover prediction.

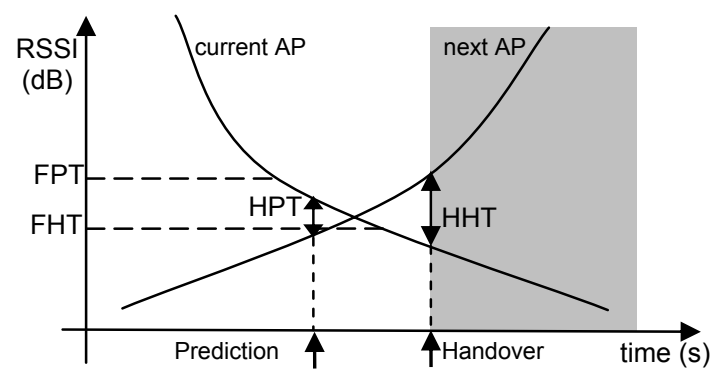

Figure 3. SP-variant prediction and handover triggers for relatively slow RSSI evolution.

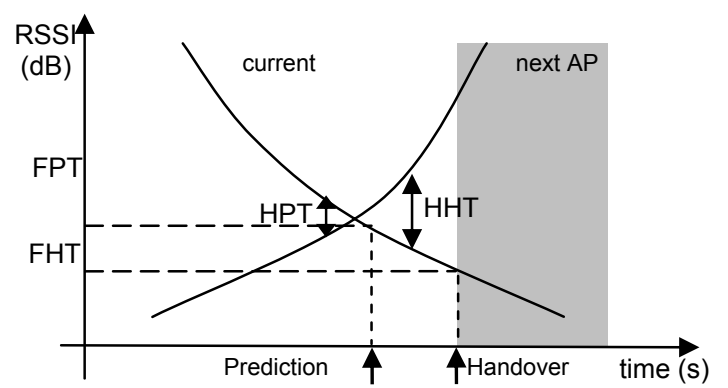

Figure 4. SP-variant prediction and handover triggers for relatively fast RSSI evolution.

\subsection{Grey Model-based RSSI Prediction}

Both variants of our predictor exploit a very simple and lightweight first-order Grey Model (GM) [12] to obtain predicted RSSI values on the basis of RSSI values monitored in the recent past. Given one visible AP and the set of its actual RSSI values measured at the client side $R_{0}=\left\{r_{0}(1), \ldots, r_{0}(n)\right\}$, where $r_{0}(i)$ is the RSSI value at the discrete time $i$, it is possible to calculate $R_{1}=\left\{r_{1}(1), \ldots, r_{1}(n)\right\}$, where

$$
r_{1}(i)=\sum_{j=1}^{i} r_{0}(j)
$$

Then, from the $\operatorname{GM}(1,1)$ discrete differential equation of the first order [7]:

$$
\frac{d r_{1}(i)}{d i}+a r_{1}(i)=u
$$

the wireless client can autonomously determine $a$ and $u$, which are exploited to obtain the predicted RSSI value $\operatorname{pr}(i)$ at discrete time $i$ according to the $\operatorname{GM}(1,1)$ prediction function [12]: 


$$
p r(i)=\left(r_{1}(1)-\frac{u}{a}\right) e^{-a k}+\frac{u}{a}
$$

The average accuracy of the RSSI prediction depends on the number of actual RSSI values $r_{0}(i)$ employed by the adopted $\operatorname{GM}(1,1)$. In principle, longer the finite input series $R_{0}$, more regular the RSSI predicted values, and slower the speed with which the $\operatorname{GM}(1,1)$ prediction anticipates the actual RSSI sequence in the case of abrupt evolution [12]. We have evaluated the performance of our predictors, as presented in the following, also while varying the number $n$ of values in $R_{0}$, without experiencing any significant improvement in the predictor performance when using $n$ values greater than 15 . For this reason, all the experimental results reported in the following will refer to the usage of $R_{0}$ sets with 15 past RSSI values.

Let us finally stress that our GM-based RSSI prediction is completely local and decentralized: each wireless client hosts its handover predictor, whose state only depends on monitored RSSI values for all APs in visibility. The only interactions needed with the associated SOMA-based proxy running in the wired infrastructure are at the moment of commanding buffer growth/reduction and proxy migration.

\section{Experimental results}

To thoroughly and quantitatively evaluate the effectiveness of our proactive buffer management solution, we have identified some performance indicators and measured them both in a simulated environment, with a large number of Wi-Fi clients roaming among a large number of APs, and in our campus deployment scenario, where 4 laptops move among the different coverage areas of 6 APs. 2 laptops are Linuxbased, while the other 2 host Microsoft Windows.NET; they alternatively exploit Cisco Aironet 350 (HP handover) and Orinoco Gold (SP handover) IEEE802.11 cards. In particular, we have considered the following performance indicators:

- Average Buffer Size $(A B S)=\frac{1}{T} \int_{0}^{T} B S(t) d t$

where $B S(t)$ is the time-varying buffer size. In other words, ABS is the time-weighted average of the amount of pre-fetched data in the buffer;

- Useful Buffered Data after handover (UBD), the available useful streaming data buffered at the proxy clone when the client associates with the new wireless cell after an handover;

- Waiting for Service after handover (WfS), the average time between client handover completion and the start of proxy-to-client data streaming in the new wireless cell;
- Successful Handover $\left(S H_{\%}\right)=\left(\frac{P H}{N H}\right) * 100$

where $\mathrm{PH}$ is the number of handovers correctly predicted by HP/SP predictors and $N H$ is the number of actual client handovers.

In general, the goal of an optimal buffer management solution is, at the same time, to minimize $A B S$ and $W f S$ and to maximize $S H_{\%}$, by maintaining a sufficiently high value for $U B D$.

We have measured the four indicators above in a challenging simulated environment where 17 APs are regularly placed in a $62 \mathrm{~m} \times 84 \mathrm{~m}$ area and RSSI fluctuation has a $3 \mathrm{db}$ standard deviation. Wireless clients follow trajectories with a randomly variable speed and with a randomly variable direction (with a Gaussian component for the standard deviation of $\Pi / 6$ ). The speed is between $0.2 \mathrm{~m} / \mathrm{s}$ and $1.2 \mathrm{~m} / \mathrm{s}$ to mimic the behaviour of walking mobile users; $F P T=72 \mathrm{db}$; $\mathrm{FHT}=80 \mathrm{db} ; \mathrm{HPT}=3 \mathrm{db} ; \mathrm{HHT}=6 \mathrm{db}$. On the average, each wireless client has the visibility of 10 APs at the same time, which represents a worst case scenario significantly more complex than actually deployed Wi-Fi networks (where no more than 5 APs are usually visible at any time and from any client position).

Table 1 reports the average results for the five performance indicators over several simulations, each simulation with about 500 handovers. The most important result is that both HP and SP predictors significantly reduce $A B S(55 \%$ and $50 \%)$ if compared with the case of a statically dimensioned nonadaptive buffer $(\mathrm{ABS}=800 \mathrm{~KB})$. This relevantly improves the memory utilization at the proxy host and reduces the network traffic due to useless prefetching. In addition, both predictors achieve a good value for $\mathrm{SH}_{\%}$, thus pointing out the satisfying performance of the GM-based handover prediction.

Client streaming players overcome cell handover with no streaming interruptions if the proxy buffer has enough useful data (not obsolete because already sent to clients by origin proxies) to fill the time interval between the end of the client-side buffer and the completion of proxy-based session re-establishment in destination cells. Since in the worst case the rebinding process lasts $2 \mathrm{~s}$ and client-sided buffers run out during communication-level handover, $U B D$ should be greater than $1 \mathrm{Mbps} * 2 \mathrm{~s}=250 \mathrm{~KB}$. $U B D$ has demonstrated to be much greater than that threshold with both the proposed predictors.

Finally, the table reports experimental results for $W f S$ in two different conditions, with and without successful prediction. In the case of correct handover prediction, a proxy with useful pre-fetched data is ready to start streaming provisioning to its client just at the completion of communication-level handover; only the time to locally re-establish the client-to- 
proxy connection is needed (about $0.2 \mathrm{~s}$ ). On the contrary, in the case of unsuccessful prediction (wrongly predicted cell or insufficient pre-fetched data at the proxy due to anticipated migration/late buffer fulfillment) a client has to discover the proxy unavailability/unsuitability, to request a new proxy/buffer, to wait for proxy/data movement, and finally to wait for service re-binding (more than $4 \mathrm{~s}$ ).

In addition to simulations, we have evaluated the adaptive buffer performance in the field, by using the implemented middleware prototype and by moving 4 client laptops among the campus wireless localities during streaming provisioning. Even if the number of considered handovers is largely lower than in the simulation case (and, thus, less relevant from the statistical point of view), in-the-field performance results confirm the ones obtained in the simulated environment. In particular, prototype-based $A B S, S_{\%}$, $W f S$ and $U B D$ have demonstrated to be, on the average, better than simulation-based ones. This is mainly due to the lower number of APs in the prototype case and the consequently simpler handover prediction. However, we have experienced a significant degradation of prototype-based performance indicators in the case of extreme RSSI fluctuations, e.g., when a client follows a trajectory in strict proximity of relevant obstacles, such as the reinforced concrete walls of our campus buildings.

Further simulation/prototype experimental results, the prototype code and additional details about its implementation are available at [8].

\begin{tabular}{|c|c|c|c|c|c|}
\hline Predictor & ABS (KB) & SH$_{\%}$ & \multicolumn{2}{|c|}{ WfS (s) } & UBD (KB) \\
\hline HP & 360 & 83.3 & 0.17 & 4.64 & 499.2 \\
\hline SP & 400 & 88.0 & 0.24 & 4.06 & 540.8 \\
\hline
\end{tabular}

Table 1. Adaptive buffering performance results when using either the HP predictor or the SP one.

\section{Related Work}

Some recent research activities have addressed position prediction in wireless networks, most of them by proposing solutions based on either the estimate of current position/speed or usual movement patterns. [13] predicts future location/speed by exploiting a dynamic Gauss-Markov model applied to the current and historical movement data. [14] bases its trajectory prediction on trajectories followed in the recent past and on the spatial knowledge of the deployment environment, e.g., by considering admissible path databases. Note that exploiting these position prediction solutions as the basis for handover prediction requires full knowledge of AP coverage area maps. In addition, in open and extremely dynamic scenarios, with medium/short-range wireless connec- tivity, user mobility behaviors change very frequently and irregularly, thus making user habits-based handover predictions almost inapplicable.

Only a few researches have already investigated RSSI prediction. [15] predicts future RSSI values by using a retroactive adaptive filter to mitigate RSSI fluctuations; the device handover is commanded when the difference between the current and the predicted RSSI values is greater than a threshold. [16] exploits GM to decide when to trigger the communication handover by comparing RSSI predictions with average and current RSSI values. However, both [15] and [16] apply RSSI prediction to improve communication-level handover, e.g., to reduce unnecessary bouncing, and not to predict the client movements for streaming buffer adaptive management.

Adaptive buffer management to avoid streaming suspension is a consolidated research area in traditional fixed distributed systems. [17] proposes proxysided adaptive buffering to decouple server-to-proxy and proxy-to-client streaming, in order to improve bandwidth utilization and to reduce client-perceived streaming fluctuations. In [18] proxies work to provide continuous streaming regardless to proxy-toserver link fault by pre-fetching data as much as possible. [19] exploits proxies to feedback servers about the suitability of increasing/decreasing transmission rate when buffers are almost empty/full. [20] proposes proxy-sided buffers to reduce initial delay and client-sided buffer size. [21] deploys a buffer at both the client side and the proxy one: the goal is to minimize connection periods instead of providing continuous services without disconnections. Moreover, it performs proactive relocation of proxy buffers by exploiting a path prediction algorithm based on the history of past movements.

To the best of our knowledge, our middleware is definitely original in providing proxy-sided adaptive buffer management for continuous services that exploits a lightweight, portable, and completely decentralized handover prediction solution, only based on RSSI data.

\section{Conclusions and On-going Activities}

The exploitation of mobile middleware proxies that work over the fixed network on behalf of their resource-constrained clients is demonstrating its suitability and effectiveness in the WI, especially when associated with handover prediction. Handover prediction can enable the proactive performing of service/middleware management operations to maintain session continuity in the provisioning of personalized services, independently of runtime client roaming. In particular, handover prediction can help in realizing 
novel adaptive buffering solutions that optimize buffer size and pre-fetching depending on the expected handover probability. The work of design, implementation, and experimental evaluation of our solution prototype has shown that our prediction-based proxy-sided adaptive buffering can preserve streaming continuity with limited requirements on wireless device memory capabilities. In addition, our buffering solution, specifically developed for mobile proxybased middleware for multimedia streaming, has a general applicability to any class of WI services that can potentially benefit from service content prefetching close to the client terminal access localities.

The promising performance results already obtained are stimulating further related research activities. We are experimenting alternative handover prediction techniques based on either higher-level GM models or the GM application to Ekahau-provided estimates of client positions (not directly to RSSI data) [22]. The goal is to evaluate whether increasing the complexity of the prediction model can significantly improve the prediction quality, thus justifying the replacement of the currently adopted $\operatorname{GM}(1,1)$ model, which is extremely simple and lightweight.

\section{Acknowledgements}

Work supported by the MIUR FIRB WEB-MINDS and the CNR Strategic IS-MANET Projects.

\section{References}

[1] M. S. Corson, J. P. Macker, V. D. Park, "Mobile and Wireless Internet Services: Putting the Pieces Together, IEEE Communications, Vol. 39, No. 6, June 2001.

[2] W. Stallings, Wireless Communications and Networks, Pearson Education, Aug. 2001.

[3] P. Ramanathan, K.M. Sivalingam, P. Agrawal, S. Kishore, "Dynamic Resource Allocation Schemes during Handoff for Mobile Multimedia Wireless Networks", IEEE Journal on Selected Areas in Communications, Vol. 17, No. 7, July 1999.

[4] S. Saha, M. Jamtgaard, J. Villasenor, "Bringing the Wireless Internet to Mobile Devices", IEEE Computer, Vol. 34, No. 6, June 2001.

[5] K. Curran, G. Parr, "A Middleware Architecture for Streaming Media over IP Networks to Mobile Devices", IEEE Int. Conf. Wireless Communications and Networking (WCNC), Mar. 2003.

[6] P. Bellavista, A. Corradi, C. Stefanelli, “Applicationlevel QoS Control and Adaptation for Video on Demand", IEEE Internet Computing, Vol. 7, No. 6, Nov.-Dec. 2003.

[7] P. Bellavista, A. Corradi, "A QoS Management Middleware based on Mobility Prediction for Multimedia Service Continuity in the Wireless Internet", IEEE
Int. Symp. on Computers and Communications (ISCC), July 2004.

[8] SOMA Smart Buffer - http://lia.deis.unibo.it/Research/SOMA/SmartBuffer/

[9] P. Bellavista, A. Corradi, R. Montanari, C. Stefanelli, "Context-aware Middleware for Resource Management in the Wireless Internet", IEEE Trans. on Software Engineering, Vol. 29, No. 12, Dec. 2003.

[10] H. Velayos, G. Karlsson, "Techniques to Reduce IEEE 802.11b MAC Layer Handover Time", Technical Report TRITAIMIT-LCN R 03:02, KTH, Stockholm, Sweden, Apr. 2003.

[11] P. Bellavista, A. Corradi, "Mobile Middleware Solutions for the Adaptive Management of Multimedia QoS to Wireless Portable Devices", $9^{\text {th }}$ IEEE Int. Workshop Object-oriented Real-time Dependable Systems (WORDS'03F), Oct. 2003.

[12] J.L. Deng, "Introduction to Grey Theory", The Journal of Grey System, Vol. 1, No. 1, 1989.

[13] B. Liang, Z.J. Haas, "Predictive Distance-Based Mobility Management for Multidimensional PCS Network", IEEE/ACM Transactions on Networking, Vol. 11, No. 5, Oct. 2003.

[14] H.A. Karimi, X. Liu, "A Predictive Location Model for Location-based Services", ACM Int. Workshop Advances in Geographic Information Systems (GIS), Nov. 2003.

[15] V. Kapoor, G. Edwards, R. Sankar, "Handoff Criteria for Personal Communication Networks", IEEE Int. Conf. Communications (ICC), May 1994.

[16] S.T. Sheu, C.C. Wu, "Using Grey Prediction Theory to Reduce Handoff Overhead in Cellular Communication Systems", IEEE Int. Symp. Personal, Indoor and Mobile Radio Communications (PIMRC), Sep. 2000.

[17] Chung-Ming H., Tz-Heng H., Chi-Kuang C., "A proxy-based adaptive flow control scheme for media streaming", ACM Symposium on Applied Computing (SAC), Mar. 2002.

[18] V. Mancuso, G. Bianchi, "Streaming for Vehicular Users via Elastic Proxy Buffer Management" IEEE Communications, Vol. 42, No. 11, Nov. 2004.

[19] Dong-Hoon N., Seung-Kyu P., "Adaptive Multimedia Stream Service with Intelligent Proxy", International Conference on Information Networking (ICOIN-15), Jan. 2001.

[20] Lei H., Uwe H., F. Hartung, M. Kampmann, "Proxybased TCP-friendly Streaming over Mobile Networks", Int. Workshop on Wireless Mobile Multimedia (WoWMoM), Sept 2002.

[21] S. Hadjiefthymiades, L. Merakos, "Proxies + Path Prediction: Improving Web Service Provision in Wireless-Mobile Communications", Mobile Networks and Applications (MONET), Vol. 8, No. 4, Aug. 2003.

[22] Ekahau, Inc. - The Ekahau Positioning Engine v2.1, http://www.ekahau.com/products/ positioningengine/ 